\title{
ARTículo
}

\section{Factores asociados a la respuesta de Pocillopora spp. (Anthozoa: Scleractinia) durante un proceso de restauración en la costa del Pacífico mexicano}

\author{
Factors associated with response Pocillopora spp. (Anthozoa: Scleractinia) \\ during a restoration process on the Mexican Pacific coast
}

\author{
Daniela Muñiz-Anguiano ${ }^{1}$, Manuel Verduzco-Zapata ${ }^{1}$ \\ y Marco A. Liñán-Cabello ${ }^{1 *}$
}

\begin{abstract}
${ }^{1}$ Facultad de Ciencias Marinas, Universidad de Colima, Km 19.5 Carretera Manzanillo-Barra de Navidad, Manzanillo, Colima, México. danny.maung@gmail.com, manuel_verduzco@ucol.mx, *linanmarco@ hotmail.com

Abstract.- During a restoration process of Pocillopora spp., using opportunity corals (CO) several biotic attributes were assessed at two sites bi-monthly over a year and concentrations of mycosporin type amino acids (MAAs) and levels of chlorophyll a (Chl a) were determined. Specifically, in the last sampling analysis it was performed an analysis of malonaldehyde (MDA) and the RNA/ DNA and protein/DNA relationships were calculated, as an index of oxidative stress and transcriptional activity in symbiont and coral tissue of all morphotypes. At Carrizales site (SC) the coral clusters (RC) used as a method of restoration, promoted the integration of $\mathrm{CO}$ showing signs of recovery and high survival rates (90\%); however, in Juluapan site (SJ), a lack of response to biotic and molecular indicators associated with recovery was observed. Therefore, $\mathrm{CO}$ from Carrizales were not able to acclimate to a place where different conditions prevail. High concentrations of $\mathrm{Chl}$ a, AAMs and a high RNA/DNA ratio were characteristic of a healthy condition of coral which in turn, were associated with higher specimen's survival and restoration.
\end{abstract}

Key words: Restoration, Pocillopora, calcareous algae, coral, oxidative stress

Resumen.- Durante un proceso de restauración de Pocillopora spp., usando corales de oportunidad (CO), fueron evaluados diversos atributos bióticos en dos sitios a lo largo de un año, de manera bimensual fueron determinadas las concentraciones de aminoácidos tipo micosporina (AAMs) y niveles de clorofila a $(\mathrm{Cl}$ a). De manera específica, en el último muestreo se realizó un análisis de malonaldehido (MDA) y se calcularon las relaciones ARN/ADN y proteína/ADN como indicadores de estrés oxidativo y actividad trascripcional en simbionte y cnidario de todos los morfotipos. En sitio Carrizales (SC) la técnica de restauración de ramilletes coralinos (RC) promovió la integración de COs registrando signos de recuperación y altas tasas de supervivencia (90\%); no obstante, en el Sitio Juluapan (SJ), se observó una falta de respuesta a los indicadores bióticos y moleculares asociados a la recuperación. Por lo tanto, los CO provenientes de Carrizales no fueron capaces de aclimatarse a un sitio en el que imperan distintas condiciones ambientales. Altas concentraciones de $\mathrm{Cl}$ a, AAMs, y una alta relación ARN/ADN fueron características de una condición saludable de corales, que a su vez, estuvieron asociados a los indicadores de restauración y mejor supervivencia observada.

Palabras clave: Restauración, Pocillopora, algas calcáreas, coral, estrés oxidativo

\section{INTRODUCCIÓN}

Las comunidades coralinas son particularmente sensibles a diversos factores de estrés, pudiendo llegar a tener un nivel de sensibilidad asociada al desarrollo de las zonas costeras, el uso excesivo de recursos arrecifales y diversas actividades antropogénicas; éstos promueven el incremento de descargas con altos contenidos de nutrientes y/o carga sedimentaria en aguas costeras. Sumado a ello, estos ecosistemas deben de afrontar los efectos derivados de la variabilidad ambiental, en donde los episodios de blanqueamiento coralino derivado de las anomalías termales han promovido serias afectaciones a escala mundial (Chavanich et al. 2009).
Las acciones orientadas a la restauración como alternativa de recuperación en poblaciones coralinas impactadas, ha sido un tópico que ha causado gran expectativa e interés comercial y científico (Edwards \& Gomez 2007, Hunt \& Sharp 2014). El éxito de un proyecto de restauración depende de múltiples factores entre los que destacan: los altos costos que permitan hacerlos extensivos a grandes áreas, el tipo de especie y sustrato seleccionado, además de que la ubicación del proyecto debe garantizar el asentamiento natural de larvas, entre otros factores (Yeemin et al. 2006). En el caso particular de la costa de Colima, México, los parches coralinos asociados a punta 
Juluapan y Carrizales son comunidades que, a pesar de no ostentar gran cobertura, son ecológica y económicamente valiosos (Edwards \& Gomez 2014). En estas comunidades imperan corales hermatípicos de 3 principales géneros: Pocillopora, Porites y Pavona, siendo el primero de ellos el de mayor abundancia, representado fundamentalmente por las especies Pocillopora verrucosa, P. capitata y P. damicornis (Reyes-Bonilla et al. 2013). No obstante, estos ambientes coralinos comparten una problemática relacionada con la degradación y pérdida de cobertura coralina, como consecuencia de diversas presiones de origen antropogénico (presión turística, sedimentación, sobrepesca, trasformación de la línea costera) (Liñán-Cabello et al. 2011). Dentro de los trabajos científicos orientados a la restauración de Pocillopora spp. en el Pacífico mexicano, en periodos anuales de monitoreo, han sido reportadas supervivencias del 95, 87 y 90,9\% (LiñánCabello et al, 2010, Tortolero-Langarica et al. 2014, Figueroa-Camacho \& Nava 2016, respectivamente). No obstante, actualmente el estudio de los procesos fisiológicos asociados a especímenes coralinos bajo un esquema de restauración, enfrenta retos por resolver que van desde la identificación de los efectos de la diversidad genotípica en las poblaciones coralinas asociadas a los ecosistemas de arrecifes que ellos soportan (Baums 2008), hasta el reconocimiento de indicadores bioquímicos de mayor sensibilidad a ser considerados dentro de un esquema de monitoreo (Liñán-Rico \& Cárdenas-Conejo 2013).
El objetivo del presente estudio fue reconocer la conveniencia en el empleo de fragmentos coralinos disponibles en el medio natural (corales de oportunidad, $\mathrm{CO}$ ), durante un plan de restauración y evaluar las respuestas fisiológicas de Pocillopora spp. El argumento hipotético se centra en reconocer la posible asociación de los trazadores de la intensidad metabólica celular y marcadores de estrés oxidativo, respecto a los indicadores de salud coralina de Pocillopora spp. No obstante, estas relaciones podrían llegar a ser influidas por factores de naturaleza biótica y abiótica ocurridas durante un año de iniciada la restauración.

\section{MATERIALES Y MÉTODOS}

\section{Área de ESTUdio}

El presente estudio se realizó en las comunidades coralinas Carrizales $\left(19^{\circ} 05^{\prime} 50.90^{\prime \prime} \mathrm{N}-104^{\circ} 26^{\prime} 15.68^{\prime \prime O}\right)$ y Juluapan $\left(19^{\circ} 05^{\prime} 50.80^{\prime \prime} \mathrm{N}-104^{\circ} 23^{\prime} 45.966^{\prime O} \mathrm{O}\right)$ en la costa de Manzanillo, Colima, México (Fig. 1). Para identificar los sitios para restauración, se consideraron los siguientes criterios: proximidad a comunidades coralinas, profundidad (menos de $6 \mathrm{~m}$ ), depresiones entre rocas de volumen entre 1,3-2,0 $\mathrm{m}^{3}$, reconociendo además un sustrato conformado con proporción aproximada de rocas pequeñas $(60 \%)$, fragmentos de coral $(30 \%)$ y arena (10\%), condiciones previamente reportadas por Liñan-Cabello et al. (2010) como propicios para restauración de Pocillopora spp.

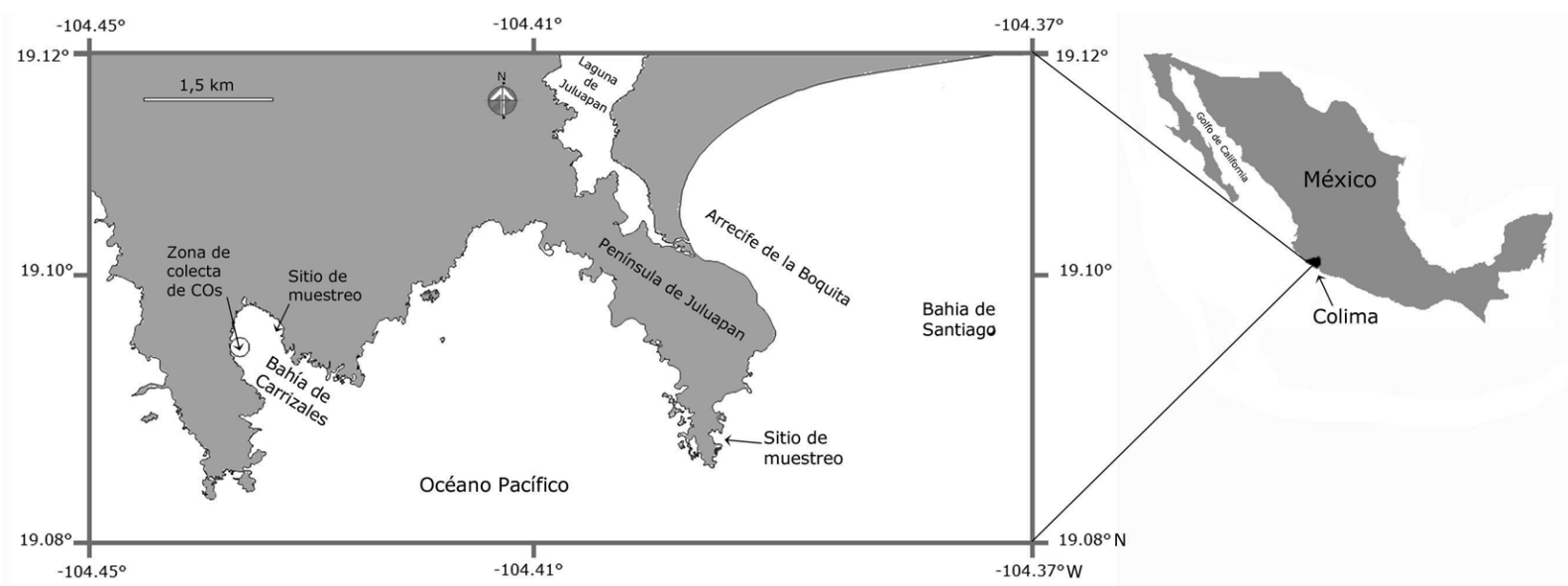

Figura 1. Localización de los sitios de estudio en la costa de Colima, México (Pacífico central mexicano) / Location of the study sites on the coast of Colima, Mexico (Mexican central Pacific) 


\section{UNIDADES EXPERIMENTALES Y TÉCNICA DE RESTAURACIÓN}

Durante enero 2012 (invierno), se colectaron 400 corales de oportunidad (CO) de Pocillopora spp. Los CO son fragmentos coralinos generados naturalmente en el arrecife y/ o como consecuencia de fragmentación incidental promovida por factores antropogénicos (Edwards \& Gómez 2007). La colecta fue realizada a profundidades entre 3-6 m de la Bahía Carrizales. Los CO seleccionados fueron entre $8-15 \mathrm{~cm}$ de largo señalado como el tamaño óptimo para técnicas de restauración (Edwards \& Gomez 2007). Los CO exhibían una palidez del tejido, entre los tonos B-E y brillo/saturación 2-3 referida en la tarjeta CoralWatch $(\mathrm{CW})$ propuesta por Siebeck et al. (2006), y empleada en otros reportes (Siebeck et al. 2008, Liu et al. 2013), ya que es considerada como método no destructivo basado en el color de los corales, útil en el monitoreo in vivo del blanqueamiento coralino. La técnica empleada para restauración fue a partir de ramilletes coralinos (RC) empleada previamente como método de restauración en la zona de estudio por Liñán-Cabello et al. (2010). Entre 9 y 10 CO fueron usados para integrar un RC, dicha integración se realizó a partir del empleo de cinchos de plástico con el fin de brindar mayor estabilidad. Cada RC fue consecutivamente marcado a partir de un cincho periférico, al cual se le integró una etiqueta, que además sirvió como referencia para evaluar la tasa de crecimiento mensual. Una vez constituidos los RC, se conformó un parche coralino (PC) de 22 RC ( 200 fragmentos coralinos), integrado entre sí con cinchos plásticos, para mejorar su estabilidad integral al fondo. El PC fue conducido a cada sitio de restauración con las características anteriormente referidas. Los PC fueron dispuestos en 2 zonas de estudio: Sitio Carrizales (SC) y Sitio Juluapan (SJ). Se eligió además un control natural $(\mathrm{CN})$, la cual consistió en un montículo coralino fijo y de mayor proximidad posible donde se presentaban especímenes de $P$. verrucosa, $P$. capitata y $P$. damicormis, como especies de mayor abundancia en las comunidades coralinas objeto de estudio (Reyes-Bonilla et al. 2013); estas colonias exhibieron tonos entre B-E y Brillo de saturación 5-6 de acuerdo a la escala CW, sin mostrar daños aparentes por enfermedad ni daños físicos por contacto.

\section{Monitoreo}

El monitoreo de atributos morfométricos y poblacionales se realizó cada dos meses durante un periodo anual (invierno 2012-invierno 2013), al igual que la toma de muestras para análisis de aminoácidos tipo micosporina (AAMs) y Clorofila $a(\mathrm{Cl} a)$, en los diferentes morfotipos: corales de oportunidad (CO), Restaurados de Carrizales (ReC), Restaurados de Juluapan (ReJ), Control Carrizales (NC), Control Jupuapan
(NJ). Con la finalidad de evaluar la condición metabólica instantánea y el estado de estrés, en el último muestreo (invierno 2013) se realizó el análisis de malonaldehido (MDA) como indicador de daño celular promovido por lipoperxidación lipídica y las relaciones ARN/ADN y proteína/ ADN como indicadores de actividad trascriptómica, en tejido de cnidario y simbionte de los morfotipos. Mediante buceo autónomo se midieron in situ cada barra coralina de un RC previamente etiquetado, obteniendo el largo y ancho, usando un medidor tipo vernier, y se registraron los umbrales de coloración con la tarjeta $\mathrm{CW}$ en cada RC. Adicionalmente, se realizaron diversos indicadores del estatus de la comunidad coralina (abundancia de algas, peces, invertebrados y esponjas), con base en los criterios propuestos por Global Coral Reef Monitoring Network (GCRMN, Hill et al. 2004), y referidos a 5 niveles de afectación: nivel 1: condición deseada, 2: presenta un patrón ligero de disgregación respecto a condición deseable (factor de afectación de 10-20\%), 3 : visiblemente disgregado o desproporcionado respecto a la condición deseable (factor de afectación de 20-40\%), 4: considerablemente afectado (factor de afectación de 40$90 \%), 5$ : totalmente afectado respecto a condición deseable (factor de afectación $>90 \%$ ). Se hizo además un registro fotográfico y videos submarinos bimensuales mediante una cámara Sony® R modelo MPK-WD, a lo largo y ancho de la cobertura en el sitio implantado.

Se registró la temperatura y salinidad del agua con un CTD (Sea Bird SBE 19 plus V2) en cada monitoreo entre 3 y $4 \mathrm{~m}$ de profundidad. Al mismo tiempo, se tomaron muestras de agua para análisis de nutrientes $\left(\mathrm{NO}_{-2}+\mathrm{NO}_{-3}, \mathrm{NH}_{4}^{+}, \mathrm{PO}_{4}^{3} \mathrm{y}\right.$ $\mathrm{SiO}_{2}$ ), que fueron analizadas en laboratorio mediante el autoanalizador de flujo segmentado (Skalar San Plus II, con una precisión de $0,001 \mu \mathrm{M}$ ), de acuerdo a los métodos referidos por Strickland \& Parsons (1972). Adicionalmente y a partir de los compuestos nitrogenados referidos, se calculó el nitrógeno inorgánico disuelto (DIN, por sus siglas en inglés). En ambas estaciones, se colocaron sensores de temperatura (HOBO Shuttle Water Proof mod. U22-001), con un protocolo de lectura de tres mediciones por día.

Para los parámetros bioquímicos, en cada muestreo se tomaron 3 fragmentos coralinos $(3-4 \mathrm{~cm})$ asociados a cada PC, en especímenes control de cada sitio y en COs existentes en la zona de colecta original como indicadores del estado basal. Las muestras se colocaron en bolsas de plástico y se mantuvieron a $4{ }^{\circ} \mathrm{C}$ durante el transporte al laboratorio, posteriormente se almacenaron a $-85^{\circ} \mathrm{C}$ hasta el análisis correspondiente. 


\section{Aminoácidos tiPo micosporina (AAMs) y Clorofila $a\left(\mathbf{C L}_{\mathbf{L}} a\right)$}

La concentración de AAMs, se determinó de acuerdo al procedimiento propuesto por Shick et al. (1992), en breve: los especímenes de coral una vez limpiados de epifitos, fueron triturados y extraídos a $4^{\circ} \mathrm{C}$ en $5 \mathrm{ml}$ de metanol al $100 \%$ gradoHPLC. Las muestras fueron centrifugadas por $5 \mathrm{~min}$ a 10,000 g y el sobrenadante fue decantado. La cuantificación de AAMs se evaluó a partir del pico de mayor absorbancia (325 nm) en un espectrofotómetro Lambda, usando el coeficiente de extinción molar $(36,200)$ referido por Bandaranayake (1998). Las concentraciones de AAMs fueron expresadas en gramos de proteína (nmol / g proteína). La concentración de Clorofila $a(\mathrm{Cl} a)$ fue determinada espectrofotométricamente (Pearson et al. 1984) usando un espectrofótometro Perkin Elmer Lambda 35.

\section{Malonaldehido (MDA), Relación ADN/ARN Y PROTEÍNA/ADN}

Para el cálculo de MDA, proteína y ácidos nucleicos se realizó la extracción de tejido de acuerdo al método Waterpik ${ }^{\mathrm{TM}}$ (Johannes \& Wiebe 1970) en breve: se removió el tejido con aire comprimido y el extracto fue depositado en tubos de 1,5 $\mathrm{ml}$, se centrifugaron a $5000 \mathrm{rpm}$ por $5 \mathrm{~min}$ a $4{ }^{\circ} \mathrm{C}$, el sobrenadante fue sonicado por $30 \mathrm{~s}$ por 6 pulsos de $5 \mathrm{~s}$ y recentrifugado; de esta manera se obtuvo el simbionte. El pellet se re-suspendió en un buffer de fosfatos $(50 \mathrm{Mm}$ de fosfato de potasio, $1 \mathrm{Mm}$ EDTA), se sonicó por $30 \mathrm{~s}$ en 6 pulsos de $5 \mathrm{~s}$, finalmente se centrifugó a 12,000 rpm por $15 \mathrm{~min}$ para la obtención del cnidario. Una vez obtenida cada fase de separación, las muestras se revisaron microscópicamente, a fin de eliminar el excedente de mucosidades y/o nematocistos presentes en las zooxantelas sedimentadas, de ser así, se procedió a limpiar estos productos celulares con agua de mar filtrada y esterilizada. Las muestras fueron analizadas el mismo día de la extracción de tejido para cada parámetro. Para la estimación de MDA se empleó el Kit TBARS (cat. No. 10009055, Cayman Chemical Company), con fundamento en la reacción entre MDA y TBARS a temperaturas altas (90$100^{\circ} \mathrm{C}$ ) y condiciones ácidas, medidas colorimétricamente a $550 \mathrm{~nm}$. Los datos fueron expresados en pM $\mu$ g-proteína ${ }^{-1}$. Los ácidos nucleicos fueron purificados y cuantificados usando una matriz de adhesión de ácidos nucleicos (Karp 2010). En particular, se utilizó un kit comercial AllPrep ${ }^{\mathrm{TM}}$ DNA/RNA/ Protein Kit (QIAGEN Inc., Valencia, CA, USA), que permite la unión selectiva de ADN genómico, del mismo modo la separación de ARN total se realizó bajo el principio RNeasy® y la precipitación química de proteínas totales se realizó en el mismo tejido. La concentración de $\mathrm{ARN}$, ADN y proteínas se determinó para cada precipitado, según el protocolo (QIAGEN Inc., Valencia, CA, USA), midiendo su absorbancia a $260 \mathrm{~nm}$, y se realizaron diluciones de buffer ARN y ADN. Para cuantificar la proteína total en los corales se empleó el Kit Coomassie ${ }^{\circledR}$ Protein Assay Reagent (Pierce, Thermo Fisher Scientific Inc., Rockford, IL, USA).

Se determinó la normalidad y homocedasticidad de los datos, se aplicó la prueba Mann-Whitney en los datos de morfometría para evaluar al final del periodo de restauración, posibles diferencias significativas entre los dos sitios objeto de estudio, estableciéndose como variables dependientes el largo y ancho. En el caso de parámetros moleculares se aplicaron pruebas de ANDEVA de una vía, a fin de determinar posibles diferencias significativas en las concentraciones $\operatorname{de} \mathrm{Cl} a$, AAMs, del tejido zooxantelar (variables dependientes), respecto a los diferentes morfotipos (variable independiente) presentes en cada estación del año. Con el propósito de evaluar la condición metabólica instantánea y el estado de estrés, en el último muestreo se realizó un ANOVA de dos vías, a fin de identificar posibles diferencias en las relaciones ARN/ADN, proteína/ADN y concentración de MDA como variables dependientes, considerandoel sitio de muestreo y el origen del tejido (cnidario o simbionte) como variables independientes. Todas las pruebas se realizaron empleando el Software STATISTICA® versión 8. Los datos se expresaron como medias \pm desviación estándar, en todos los casos se consideró un nivel de significancia de $P=$ 0,05 .

\section{Resultados}

\section{Cambios estacionales en restauración}

El promedio de temperaturas en primavera fueron de 23,88 $\pm 1,68$ y $23,25 \pm 1,5^{\circ} \mathrm{C}$, para SC y SJ respectivamente; durante el verano el valor más alto fue de $29,5^{\circ} \mathrm{C}$ en Carrizales, destacando que en una semana de abril se registró una disminución hasta $15,81^{\circ} \mathrm{C}$. En Juluapan la temperatura más alta ocurrió en otoño $\left(30,79^{\circ} \mathrm{C}\right.$, Tabla 1$)$. En cuanto a los niveles de sensibilidad de indicadores relacionados con el status de la comunidad coralina durante invierno 2012, prevaleció en un nivel 2 en ambos sitios, considerándose ligeramente afectado. No obstante, en el invierno del 2013, en el sitio Carrizales los indicadores referían una condición deseable, mientras que en $\mathrm{SJ}$ se presentaron condiciones de sensibilidad. En este sitio se observaron señales de bioerosión presentadas principalmente por mordeduras de peces, presencia de caracoles coralívoros y crecimiento algal (Tabla 2, Fig. 2a y b). Por otro lado, en el tiempo inicial (To), la frecuencia en porcentaje del umbral de coloración de la tarjeta $\mathrm{CW}$, fue de 2 en ambos sitios, no obstante, en SC en invierno 
Tabla 1. Promedio de temperatura ( $n=90$ registros por mes) $\pm D E$ y concentración de nutrientes ( $\mu$ moles) en Carrizales y Juluapan durante primavera, verano y otoño 2012 e invierno 2013, $n=2$ por muestreo, DIN: Nitrógeno inorgánico disuelto / Average temperature ( $n=90$ records per month) \pm SD and nutrient concentration ( $\mu$ moles) in Carrizales and Juluapan Spring, Summer and Autumn 2012 and Winter 2013, n= 2 sample, DIN: dissolved inorganic nitrogen

\begin{tabular}{lcccc}
\hline \multicolumn{1}{c}{ Parámetro } & Primavera & Verano & Otoño & Invierno \\
\hline Carrizales & & & & \\
Temperatura $\left({ }^{\circ} \mathrm{C}\right)$ & $23,8 \pm 3,8$ & $29,5 \pm 0,6$ & $29,2 \pm 0,8$ & $25,8 \pm 2,5$ \\
$\mathrm{DIN}(\mu \mathrm{M})$ & 6,15 & 4,34 & 4,50 & 5,94 \\
$\mathrm{PO}_{4}(\mu \mathrm{M})$ & 3,51 & 0,62 & 0,68 & 0,55 \\
$\mathrm{SiO}_{2}(\mu \mathrm{M})$ & 7,76 & 5,83 & 5,06 & 6,29 \\
$\mathrm{Juluapan}$ & & & & \\
Temperatura $\left({ }^{\circ} \mathrm{C}\right)$ & $23,2 \pm 1,5$ & $29,8 \pm 0,3$ & $30,7 \pm 0,6$ & $26,4 \pm 1,5$ \\
$\mathrm{DIN}(\mu \mathrm{M})$ & 7,46 & 5,12 & 4,39 & 4,64 \\
$\mathrm{PO}_{4}(\mu \mathrm{M})$ & 3,62 & 0,49 & 0,74 & 0,58 \\
$\mathrm{SiO}_{2}(\mu \mathrm{M})$ & 7,07 & 6,90 & 5,24 & 5,97 \\
\hline
\end{tabular}

Tabla 2. Valores promedio de parámetros morfológicos e indicadores de salud durante invierno 2012 y 2013 en ambas áreas de estudio. Valor 1: Condición deseada, 2 : ligera disgregación respecto a la condición deseable (que afecta a 10$20 \%$ ), 3: considerable disgregación respecto a la condición deseable (afectando entre 20-40\%), 4: afectada significativamente (afecta a 40-90\%), 5: totalmente afectada (afectación $>90 \%$ ). Literales diferentes representan diferencias significativas $(\mathbf{P}<\mathbf{0 , 0 5})$ / Average values of morphometric parameters and health indicators during Winter 2012 and 2013, in both study areas. Value 1: desired condition, 2: slight disintegration or disproportionate to the desirable condition (affecting 10-20\%), 3: visibly disintegrated or disproportionate (affecting 20-40\%), 4: significantly affected (affecting between 40-90\%), 5: totally affected (affecting $>90 \%$ ). With different literal values indicate significant difference $(P<0.05)$

\begin{tabular}{lcc}
\hline \multicolumn{1}{c}{ Parámetro } & Carrizales & Juluapan \\
\hline Número de fragmentos coralinos/sitio & 200 & 200 \\
Número de ramilletes coralinos /sitio & 22 & 22 \\
Crecimiento (cm/mes), promedio. (n= 125) & $0,081 \pm 0,01^{\mathrm{a}}$ & $0,078 \pm 0,01^{\mathrm{a}}$ \\
Coral vivo final (\%) (Después de 1 año) & $90 \%$ & $40 \%$ \\
Invierno 2012 & & \\
Abundancia de algas & 3 & 2 \\
Abundancia de peces & 2 & 2 \\
Abundancia de invertebrados & 2 & 2 \\
Abundancia de esponjas & 2 & 2 \\
Blanqueamiento & 2 & 2 \\
Invierno 2013 & & \\
Abundancia de algas & 1 & 4 \\
Abundancia de peces & 1 & 1 \\
Abundancia de invertebrados & 1 & 1 \\
Abundancia de esponjas & 1 & 2 \\
Blanqueamiento & 1 & 4 \\
\hline
\end{tabular}



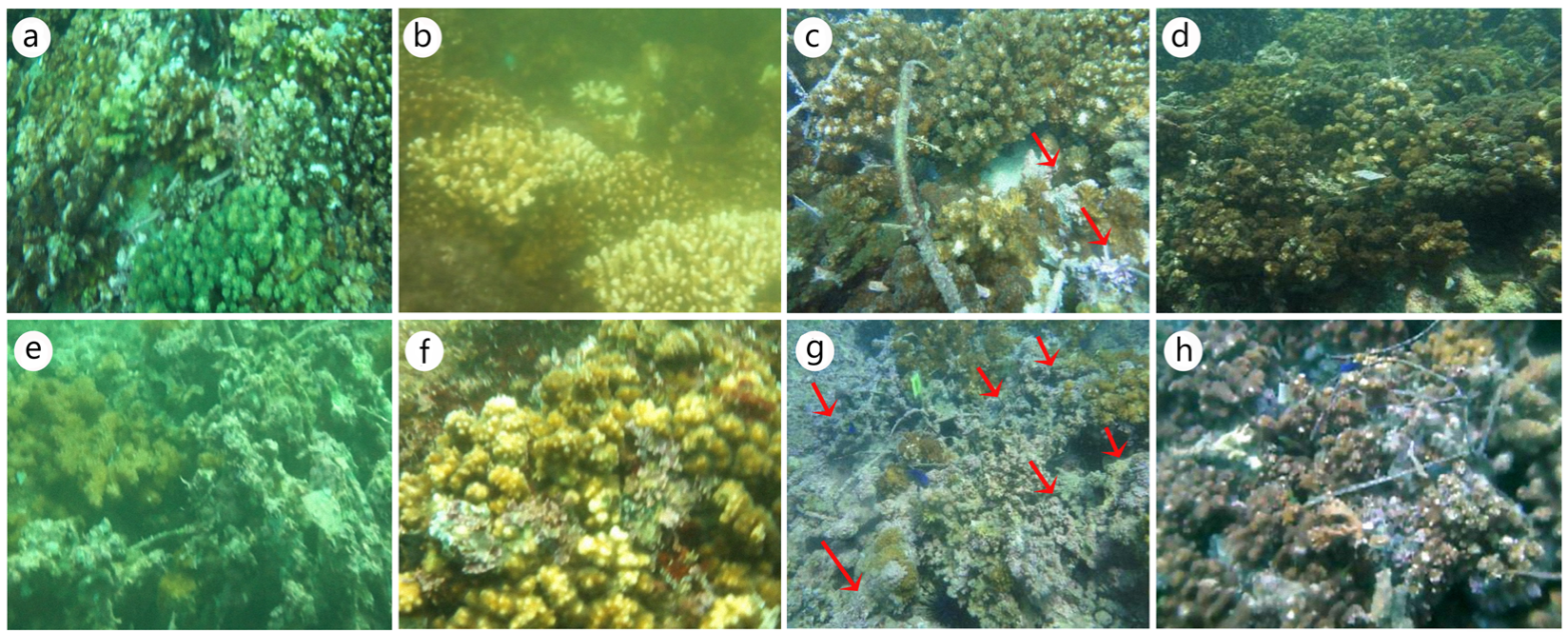

Figura 2. Algunas evidencias de los indicadores bióticos en las áreas de estudio. Sitio Carrizales: a) daño por bioerosión, b) decoloración parcial; c) presencia de algas; d) vista final invierno 2013. Sitio Juluapan: e) daño por bioerosión; f) decoloración parcial; g) presencia de algas; $h$ ) vista final invierno 2013. Las flechas señalan el crecimiento de algas / Some evidence of biotic indicators in the study areas. Carrizales site: a) bioerosion damage; b) partial discoloration; c) presence of algae; d) final view Winter 2013. Juluapan site: e) bioerosion damage; f) partial discoloration; g) presence of algae; h) Winter 2013 final view. The arrows indicate the growth of algae

a)

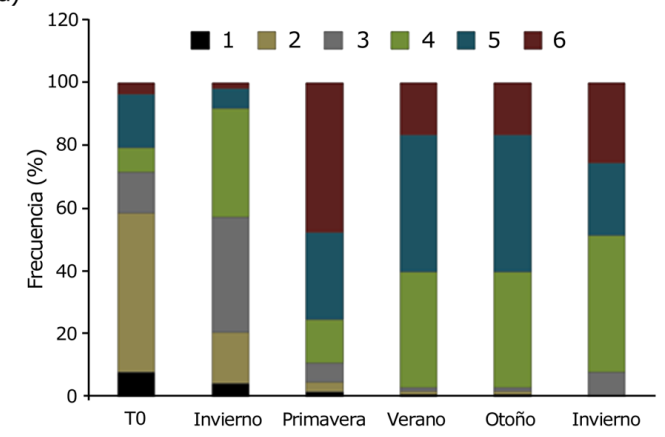

b)

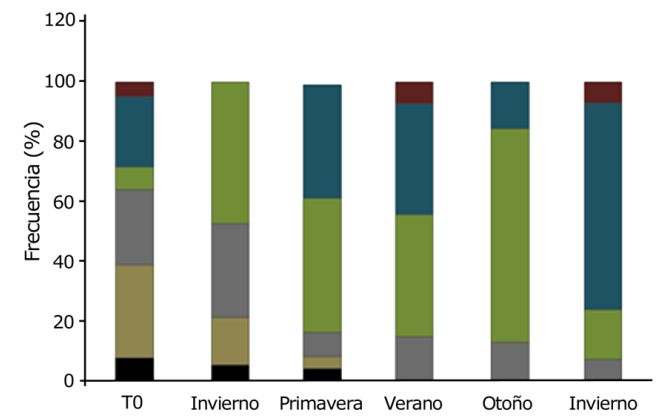

Figura 3. Representación de umbrales de coloración (CoralWatch) monitoreados en Tiempo inicial (To) y durante las 4 estaciones del año (invierno, primavera, verano, otoño 2012 e invierno 2013): a) Carrizales (SC) y b) Juluapan (S) / Representation thresholds color (CoralWatch) monitored in initial time (To) and during the 4 seasons (Winter, Spring, Summer, Autumn 2012 and Winter 2013): a) Carrizales (SC) and b) Juluapan (SJ) dominaron los umbrales 3 y 4; en primavera 6; verano y otoño de 4 y 5 (Fig. 3a). Por su parte, en SJ se obtuvo un umbral 4 durante las 4 estaciones del 2012, destacando un cambio en el invierno 2013 con umbral 5. Igualmente se observó que SC presentó mayor diversidad de umbrales de coloración con respecto a SJ (Fig. 3b). En general las concentraciones de $\mathrm{DIN}, \mathrm{PO}_{4}$ y $\mathrm{SiO}_{2}$ fueron mayores durante primavera tanto en EP como en EC. Igualmente, las menores concentraciones de este parámetro se observaron entre verano y otoño. En cuanto a la temperatura superficial se observaron valores más altos en EJ respecto a EC. En primavera, la concentración de $\mathrm{Cl} a$ fue significativamente más alta $(\mathrm{F}=33,59, \mathrm{~g} . \mathrm{l}=17, P=0,0001$; prueba de Tukey, 9,30 $\mu \mathrm{g} \mathrm{cm}^{-2}$, g.l= 16, $\left.P=0,0018\right)$ en muestras del morfotipo $\mathrm{ReC}$, mientras que en verano las concentraciones fueron significativamente mayores en especímenes asociados a $\operatorname{ReC}$ (Tukey, $3,46 \mu \mathrm{g} \mathrm{cm}^{-2}, \mathrm{~g} . \mathrm{l}=16$, $P=0,024)$ y a ReJ (Tukey, $3,38 \mu \mathrm{g} \mathrm{cm}^{-2}, \mathrm{~g} . \mathrm{l}=16, P=0,005$ ). Durante otoño no se encontraron diferencias significativas (Tukey, $\mathrm{NC}=0,31, \mathrm{ReC}=0,17, \mathrm{NJ}=0,12, \mathrm{ReJ}=0,26 \mu \mathrm{g}$ $\mathrm{cm}^{-2}$, g.l $\left.=16, P=1,00\right)$. En invierno 2013, la concentración de ReJ fue mayor respecto a ReC (Tukey, $3,52 \mu \mathrm{g} \mathrm{cm}^{-2}$, g.l.= $16, P=0,005)$.

Con relación a los AAMs, en primavera se registraron concentraciones significativamente altas $(\mathrm{F}=0,0002, \mathrm{~g} . \mathrm{l}=17$, $P=0,0001$; Tukey, 935,35 $\mathrm{nmol} \mathrm{g}^{-1}$ proteína, g.l $=18, P=$ 0,0001 ) en $\mathrm{CO}$ respecto al resto de las muestras (Fig. 4). Durante invierno 2013 la concentración más alta fue en NC de 
Carrizales (Tukey, 5767,80 nmol g ${ }^{-1}$ proteína, g.l $=18, P=$ $0,001)$ siendo significativamente mayor al resto de las muestras (Fig. 4).

\section{INDICADORES DE RECUPERACIÓN CORALINA}

De acuerdo a los registros morfométricos de fragmentos previamente marcados, se observó que la tasa de crecimiento promedio de Pocillopora spp. en restauración fue de 0,081 y $0,078 \mathrm{~cm} \mathrm{mes}^{-1}$ para SC y SJ, respectivamente (Tabla 2), sin presentar diferencias significativas $(P=0,06)$. De acuerdo a las evaluaciones in situ, análisis fotográficos y videos de cada PC al inicio y final, en Carrizales se obtuvo $90 \%$ de supervivencia de los RC asociados a cada PC, los cuales permanecieron fijos a los sustratos rocosos y coralinos circundantes, no hubo señales de palidez del tejido, se presentó gradualmente mayor abundancia de organismos bentónicos y pelágicos, dentro de los que se pudieron identificar; moluscos (Glossodoris sedna y Thais speciosa), poliquetos Spirobranchus sp., algas Corallina sp. y la esponja Haliclona caerulea; así como también, los peces: Stegastes acapulcoensis, Thalassoma lucasanum, Holacanthus passer y Microspathodon dorsalis); la mortalidad estuvo asociada básicamente al sobre-crecimiento de Corallina spp. y sedimentación. Por su parte en SJ, se registró predominancia de $S$. acapulcoensis y T. lucasanum, y una supervivencia final de 40\%. En este sitio se observó el sobre-crecimiento de algas calcáreas (Corallina sp.) (Fig. 2).
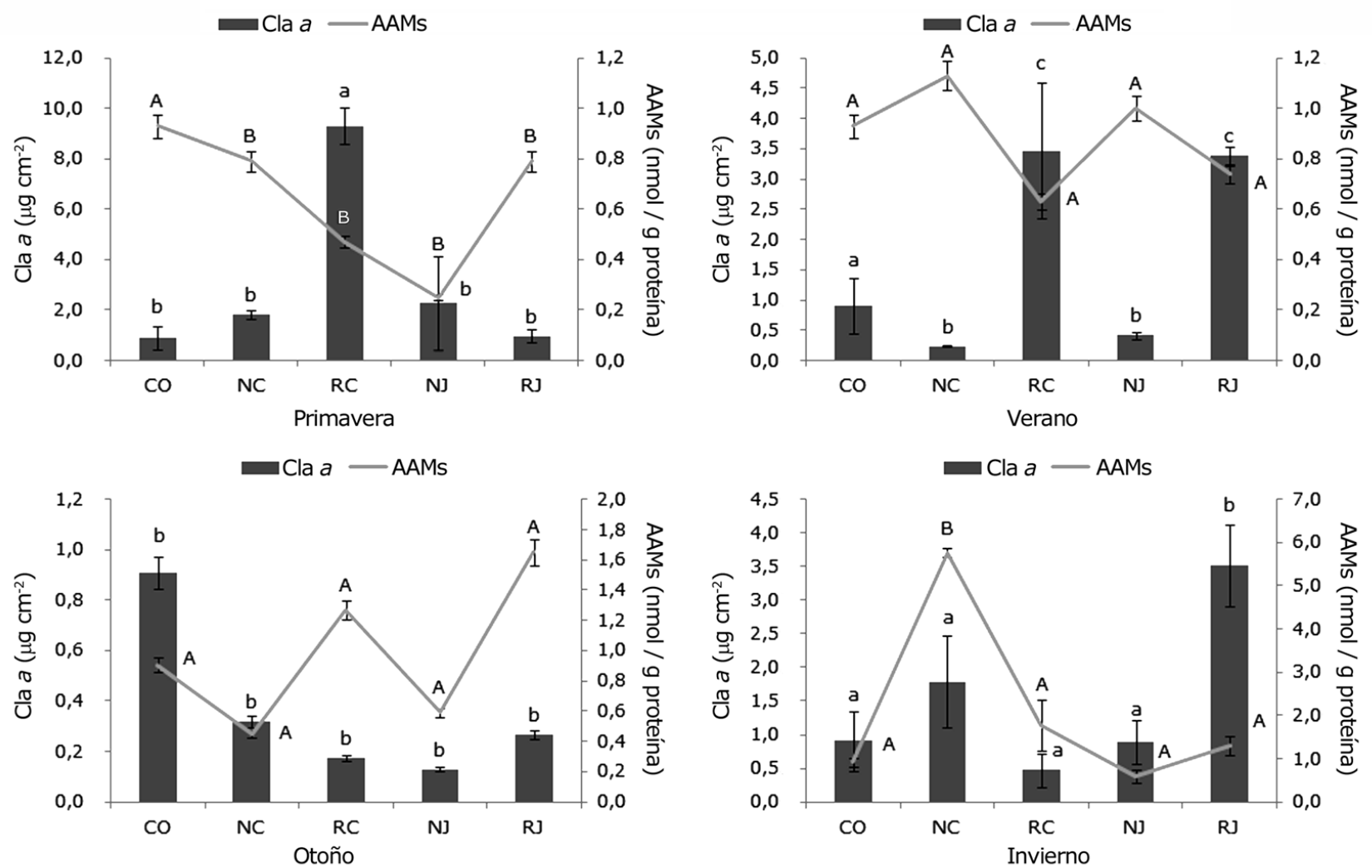

Figura 4. Valores promedio de las concentraciones de clorofila a (Cla a) y aminoácidos tipo micosporina (AAM s) en Pocillopora spp.; durante primavera, verano, otoño 2012 e invierno 2013. CO: Corales de oportunidad, NC-NJ: corales naturales de Carrizales-Juluapan (Control), ReC-ReJ: corales en restauración de Carrizales-Juluapan. Literales diferentes indican diferencias significativas, $P<0,05$. Los datos son presentados como medias $\pm D E$ / Average values of chlorophyll a concentrations (Cla a) and amino acids mycosporin type (MAAs) in Pocillopora spp.; during Spring, Summer, Autumn 2012 and Winter 2013. CO: Corals opportunity, NC-NJ: natural corals Carrizales-Juluapan (Control), ReC-ReJ: coral restoration of Carrizales-Juluapan. Different letters indicate significant differences, $P<0.05$. The data are presented as mean \pm SD 


\section{ESTRÉS Y CONDICIÓN TRASCRIPTÓMICA}

Posterior a un año de iniciada la restauración en general se distinguieron concentraciones significativamente mayores de MDA en cnidario respecto al simbionte, particularmente en COc (Tukey, 0,0015 $\mu \mathrm{M} / \mu \mathrm{g}$ proteína, g.l. $=8, P=0,037$ ); NCc (Tukey, $0,0016 \mu \mathrm{M} / \mu \mathrm{g}$ proteína, g.l $=8, P=0,020)$ y en $\mathrm{RCc}$ (Tukey, 0,015 $\mu \mathrm{M} / \mu \mathrm{g}$ proteína, g.l= $8, P=0,039$ ); (en lo sucesivo subíndices c y s representa tejido de cnidario y simbionte respectivamente); respecto a NCs. Se observó que dentro de cnidario el morfotipo RJc, se presentaron las concentraciones de MDA marginalmente significativamente más bajas (Tukey, g.l $=8, P=0,0004$, valor del estadígrafo, $P=$ 0,047) (Fig. 5). Respecto a los indicadores asociados a la actividad metabólica, se reconocieron 3 principales tendencias: a) marcada desproporción entre altas relaciones de proteína/ ADN (rango de 3,0-9,8), respecto a bajos valores en la relación ARN/ADN (rango de 0,22-0,85), (morfotipos CO, ReC, ReJ); b) menor desproporción entre los indicadores antes referidos (morfotipo NC) y c) se invierten los patrones anteriores y ahora el valor de la relación ARN/ADN (14) superó la relación proteína/ADN (morfotipo NJ) $(12,8)$. Por otro lado, cuando se analizó por separado el comportamiento de dichas relaciones por parte del simbionte y cnidario en los morfotipos control de ambos sitios, se pudo observar que tanto en NCs y NJs, la contribución del simbionte fue menor respecto al presentado en cnidario de ambos morfotipos (NCc y NJc) (Fig. 6).

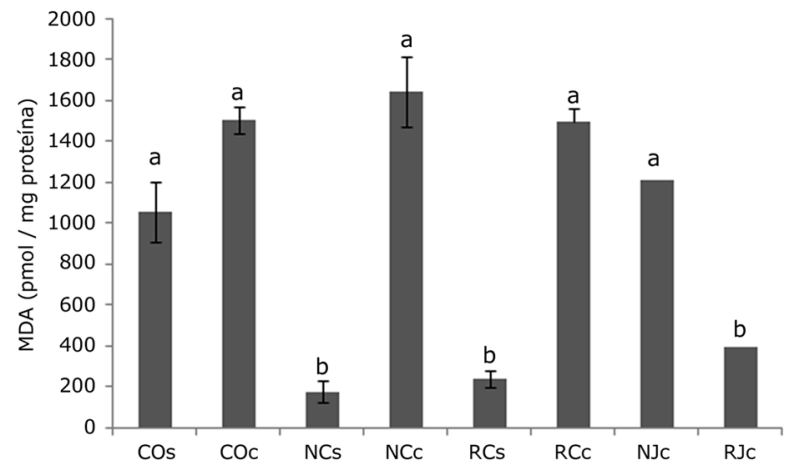

Figura 5. Comparación entre las concentraciones de MDA (pmol/ $\mathrm{mg}$ proteína) en cnidario (c). Muestras de coral de oportunidad (CO), corales naturales de Carrizales (NC), corales en restauración de Carrizales (RC), corales naturales de Juluapan (NJ) y corales en restauración de Juluapan (RJ). Las literales diferentes indican diferencias significativas, $\mathrm{P}<\mathbf{0 , 0 5}$. Los datos son presentados como medias $\pm \mathrm{DE}$ / Comparison between the concentrations of MDA (pmol / $\mathrm{mg}$ protein) in cnidaria (c). Coral samples opportunity (CO), natural corals Carrizales (NC), coral restoration Carrizales (RC), natural corals Juluapan (NJ) and coral restoration Juluapan (RJ). The different literal indicates significant differences, $P<0.05$. The data are presented as mean \pm SD

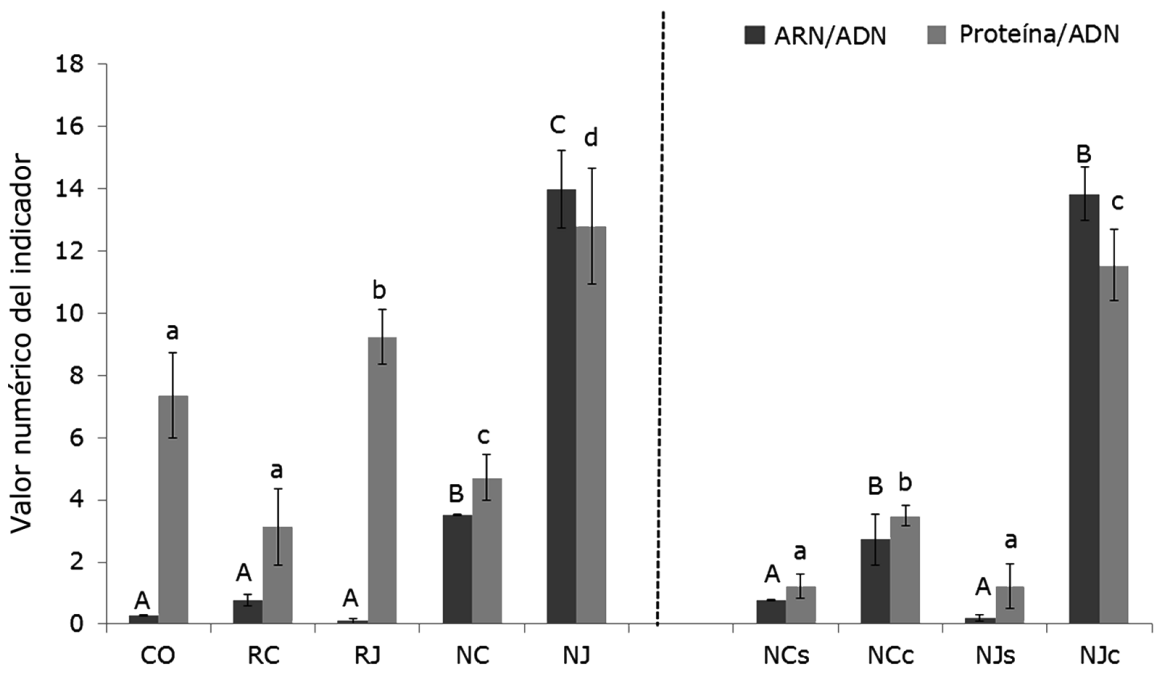

Figura 6. Relación ARN/ADN y proteína/ ADN en muestras coral de oportunidad (CO); corales naturales de Carrizales NC; corales en restauración de Carrizales ( $\mathrm{ReC}$ ), corales naturales de Juluapan ( $\mathrm{NJ}$ ) y corales en restauración de Juluapan (ReJ). Muestras cnidario (c) y muestras simbionte (s). Las literales diferentes indican diferencias significativas, $P<0,05$. Los datos son presentados como medias $\pm D E$ / RNA/DNA and protein / DNA ratios in coral samples opportunity (CO); Carrizales natural corals NC; coral restoration Carrizales (ReC), natural corals Juluapan (NJ) and coral restoration Juluapan (ReJ). Cnidario samples (c) and symbiont samples (s). The different letters indicate significant differences, $\mathrm{P}<0.05$. The data are presented as mean \pm SD 


\section{Discusión}

Las comunidades coralinas además de afrontar los eventos relacionados con la variabilidad ambiental, son particularmente sensibles a las afectaciones de origen antropogénico. Lo anterior hace necesario que en un esquema de restauración tenga a bien considerarse el entorno ambiental. En la presente investigación, la temperatura superficial promedio durante el proceso de restauración estuvo muy próxima de los niveles óptimos $\left(28,5^{\circ} \mathrm{C}\right)$ reportadas para $P$. verrucosa en el Pacífico central mexicano (Rodríguez-Troncoso et al. 2010). No obstante, durante el invierno se registró de manera excepcional una anomalía térmica que ocasionó un descenso a $15,8^{\circ} \mathrm{C}$, y posterior a ello únicamente en la estación Carrizales se presentó blanqueamiento local generalizado. Sin embargo, la recuperación observada en este sitio sin mortalidad en 3 semanas de dicho tiempo, permitió confirmar la capacidad de resiliencia característica del género Pocillopora referida frente a eventos de estrés térmico (Obura 2009).

En general en las dos estaciones de muestreo y durante el periodo de restauración fueron observados altos niveles de nutrientes. En este sentido, algunos autores refieren que bajo un efecto de enriquecimiento de nutrientes proliferarán algas y/ o pastos marinos sobre los corales, bloqueando la luz solar, inhibiendo el crecimiento o afectando las tasas de calcificación (Szmant 2002, Smith et al. 2010), produciendo así, efectos a nivel fisiológico en la relación coral-hospedero (Szmant 2002). De acuerdo a Fabricius (2005), los valores de DIN preferentemente deben estar por bajo de $1,0 \mu \mathrm{M} \mathrm{L}^{-1}$, y de $\mathrm{PO}_{4}$ total disuelto en menos de $0,1 \mu \mathrm{M} \mathrm{L}^{-1}$ para favorecer el estado fisiológico de comunidades coralinas. En este trabajo las concentraciones de DIN fueron superiores en ambas zonas a los valores antes referidos durante las diferentes estaciones del año. En un estudio reciente realizado por Liñán-Cabello et al. (2016), se refiere que el origen en las altas concentraciones de nutrientes en el parche coralino La Boquita localizado aproximadamente a $800 \mathrm{~m}$ del sitio Juluapan fue por escorrentías continentales y aportes antropogénicas de la Laguna Juluapan. Bajo este contexto, el alto contenido de nutrientes a lo largo del periodo de muestreo, y en especial durante la temporada de lluvias, pudo haber favorecido el florecimiento de comunidades algales en las comunidades coralinas estudiadas y la falta de capacidad fotosintética por parte del simbionte, posiblemente interfirió en los procesos de calcificación del coral (Anthony \& Fabricius 2000).

Con relación al crecimiento de Pocillopora existe variabilidad en los reportes de incremento de talla referidos. Prahl \& Vargas-Angel (1990) en un arrecife costero del Pacífico Colombiano reconocieron una relación de crecimiento de 0,15 $\mathrm{cm} \mathrm{mes}^{-1}$ en P. darmicornis. Tortolero-Langarica et al. (2014) reportaron durante un evento de restauración de Pocillopora spp. en las islas Marietas del Pacífico Central de México, tasas de crecimiento longitudinal y radial de 0,45 y $0,46 \mathrm{~cm} \mathrm{mes}^{-1}$, respectivamente. No obstante, niveles de incremento de talla con mayor similitud a las obtenidas en el presente trabajo $(0,078$ $0,081 \mathrm{~cm} \mathrm{mes}^{-1}$ ), fueron reportadas por Anderson et al. (2012), quienes registraron un promedio $0,054-0,120 \mathrm{~cm} \mathrm{mes}^{-1}$ en $P$. darmicornis, en comunidades del Pacífico Oriental Tropical. En general, los corales ramificados se caracterizan por su rápida extensión lineal, aunque el crecimiento es a veces moderado por la necesidad de aumentar la densidad del esqueleto, y con ello soportar fuerzas hidrodinámicas (Anderson et al. 2012). Forrester et al. (2012) señalaron que el género Pocillopora exhibe plasticidad fenotípica en respuesta a las condiciones de la dinámica oceánica, esqueleto y tamaño del pólipo. Aunque las tasas de crecimiento fueron bajas se considera que en SC, estuvieron dentro del rango aceptable sobre todo por tratarse de un proceso de recuperación de fragmentos coralinos sin mucha expectativa a sobrevivir en el medio natural. Con base en las observaciones, la presencia del alga calcárea Corallina sp., fue la causante de la mortalidad del $60 \%$ de los corales en SJ. Algunas investigaciones señalan que el sobre crecimiento de algas crustosas (a la cual pertenece la Corallina sp.) está relacionada con mayor presencia de herbívoros, pero en ausencia de ellos, la densidad algal se incrementa (Szmant 2002, Smith et al. 2010). Sin embargo, independientemente de la estación del año, en SJ, fue muy evidente que las comunidades algales fungieron como bierosionadores activos (Figs. 2, 3 y 4b). Con relación a ello, ha sido reconocido que comunidades coralinas de mediana cobertura como es el caso de Carrizales, podrían exhibir mayor predominancia y abundancia de plánulas, lo cual le permitiría responder de manera más favorable a cambios bruscos que imperan en el ambiente, respecto a otras colonias o parches coralinos pequeños con poca extensión y conexión (Pandolfi et al. 2011). Dentro de este contexto, es importante reconocer que $\mathrm{SJ}$ posee poca extensión coralina $(\approx$ $250 \mathrm{~m}^{2}$ ) alternada con sustratos rocosos, mientras que SC se extiende por más de varias hectáreas, siendo por ello SJ más limitado en cuanto al aporte de reclutas, y con ello a afrontar el efecto invasivo de algas.

Las comunidades de peces forman parte determinante en los arrecifes coralinos, de manera que el funcionamiento de dichos entornos es influido por la dinámica de grupos tróficos, controlando la estructura específica y abundancia a través de la competencia y depredación, contribuyendo así al flujo energético de los ecosistemas (Galván-Villa et al. 2011). En el presente trabajo, sólo se practicó una estimación cualitativa en cuanto a presencia de peces, no obstante, en SJ la alta 
abundancia de peces registrada fue principalmente asociada a S. acapulcoensis y T. lucasanum. Galván-Villa et al. (2011) y López-Pérez et al. (2012) resaltaron la abundancia de $S$. acapulcoensis y T. lucasanum en parches coralinos del Pacífico central mexicano. Dichos estudios coinciden que estas especies presentan una abundancia asociada con un estado de degradación en los entornos coralinos estudiados. Con base en lo anterior se puede considerar que la presencia de $S$. acapulcoensis, al ser de gran abundancia durante todas las estaciones del año en SJ, pudo haber contribuido a la pérdida de tejido coralino por efecto intenso de mordedura, efecto previamente reportado para dicha especie por Miller \& Hay (1997); teniendo por ello un efecto bioerosivo directo sobre la supervivencia del coral. Adicionalmente, la predominancia de este organismo pudo haber limitado la presencia de otros herbívoros, y con ello favoreció la proliferación y sobrecrecimiento de algas calcáreas en la comunidad coralina. En cuanto a la recuperación coralina valorada a partir del cambio en el umbral de saturación de color, los resultados de ambos sitios se asemejan a lo reportado por Liñán-Cabello et al. (2010), quienes registraron un cambio tanto en tono, como en umbral de color en el primer y segundo mes después del trasplante de corales Pocillopora spp. De acuerdo a Siebeck et al. (2006) estos cambios podrían representar un mejor estado de salud, con respecto al estado inicial de la restauración en ambos sitios. Por otro lado, se considera que la mayor diversidad de tonos en Carrizales a lo largo de las estaciones del año, posiblemente se debió a que dicho sitio por efecto de su mayor extensión, se encuentra bajo mayor influencia y abundancia de clados de Symbiodinium. Con relación a ello, se ha reconocidoque las extensiones coralinas amplias generan mayor recambio genético a nivel de simbiontes y hospederos, y como beneficio adquieren resistencia a diferentes factores de estrés (Cunning et al. 2013). Lo anterior permite reconocer que una de las desventajas de la restauración en el sitio Juluapan fue posiblemente la poca extensión coralina preliminarmente existente.

De acuerdo a los niveles determinados de MDA como indicador de daño celular por estrés oxidativo realizado al final del periodo de muestreo, en general se reconocieron valores que fueron bajos respecto a las concentraciones previamente reportadas por Liñán-Cabello et al. (2009). Particularmente, las concentraciones significativamente bajas de MDA en simbionte tanto de morfotipos naturales como de restaurados (NCS y ReS), podría ser un reflejo de una mayor capacidad antioxidante promovida por las altas concentraciones de AAMs, observadas durante la mayoría de las estaciones del año ya que este tipo de compuestos tienen su origen en tejido simbiótico (Korbee et al. 2006). De acuerdo a dicho autor, estos compuestos están relacionados con múltiples funciones fisiológicas, tales como: osmoregulación, antioxidantes, pigmentos accesorios, fotoprotectores y reservorios de nitrógeno.

Por otro lado, de acuerdo a la relación ARN/ADN, como indicador trascripcional, fue posible reconocer que en ambos morfotipos control, el simbionte exhibió valores bajos respecto a lo observado en el cnidario. Lo anterior ya había sido observado por Mayfield et al. (2011) quienes además postularon que el simbionte aporta únicamente el $10 \%$ de los trascriptos de ARN en el coral Seriatopora hystrix. En este sentido también se observó que los morfotipos NCc y NJc presentaron las mayores relaciones de proteína/ADN, lo cual no ocurrió con ninguno de los corales restaurados, y podría ser el reflejo de que, posterior a un año de iniciada la restauración, los CO no alcanzaron el estado trascripcional que caracterizó a los especímenes de control. Lo anterior representa un argumento molecular en relación con lo reconocido por Edwards \& Gomez (2007), quienes postulan que la restauración no debe ser medible en tiempos cortos, ya que los signos de adaptación más evidentes pueden llegar a ser detectados en un lapso de varios años. Por otro lado, los altos incrementos de la relación proteína/ADN y bajas relaciones de ARN/ADN en el simbionte ( $\mathrm{CO}$, y RJ), podrían estar asociados a un estado de estrés específico en estos especímenes, promovido por la sobreexpresión puntual de proteínas de choque térmico, lo cual ha sido previamente referido en Acropora muricata (Seveso et al. 2012).

Respecto a la relación ARN/ADN, ha sido reportado un rango de 0,2-3,9, en virtud a su nivel de exposición a variaciones del ambiente y/o exposición a agentes estresantes (Mayfield $e t$ al. 2013). Los valores reportados para la mayoría de los morfotipos estuvieron en este intervalo. Sin embargo, Hauri $e t$ al. (2010) determinaron valores aún más altos (3-6) que se asemejan más al obtenido en la presente investigación para NJ. De acuerdo a ello, los autores asociaron dichos valores presentados en el coral Acropora millepora a la presencia de macroalgas, las cuales estimularon la síntesis de proteínas de estrés en respuesta a cambios en el ambiente. La baja relación del indicador ARN/ADN en CO, RC y RJ, podría interpretarse como un abatimiento en la capacidad de respuesta fisiológica para responder a la variabilidad ambiental. Es importante destacar que los corales de oportunidad fueron originalmente colectados de la comunidad coralina Carrizales y al ser expuestos a una zona con otro historial ambiental, no pudieron ser capaces de aclimatizarse y con ello fueron más sensibles a la bioerosión por macroalgas. En Bahía Carrizales la técnica de ramilletes coralinos como método de restauración, durante las diferentes estaciones del año, promovió la integración de 
los corales de oportunidad, registrando signos de recuperación y alta supervivencia. Por otro lado, en el sitio Juluapan se observó una falta de respuesta a los indicadores bióticos y moleculares asociados a la recuperación. Se estima que los corales de oportunidad provenientes de Carrizales no fueron capaces de aclimatarse a un sitio en el que imperan distintas condiciones. Con base en lo determinado en la relación ARN/ ADN, fue posible distinguir un estado trascripcionalmente más activo en cnidario respecto a lo observado en tejido de simbionte.

Concentraciones altas de $\mathrm{Cl} a$, AAMs, y una relación alta de ARN/ADN fueron características de una condición saludable de corales que a su vez estuvieron asociados al estado que denotó mayor en las muestras de control, respecto a corales restaurados de mayor supervivencia, podría ser un indicador que durante el periodo de monitoreo, los especímenes restaurados aun no alcanzaban un estado fisiológico óptimo. Se recomienda la realización de estudios donde estos indicadores sean evaluados en procesos de mayor cobertura temporal.

\section{Agradecimientos}

Los autores agradecen a Ernesto Torres-Orozco y Aramis Olivos-Ortiz por las facilidades otorgadas para la obtención de parámetros fisicoquímicos, a los revisores anónimos por sus valiosas aportaciones. Igualmente se agradece a Alejandro Nuño, Joel Bracamontes-Ramírez, y Olinda Soriano, por su apoyo en las actividades de campo.

\section{LITERATURA CITADA}

Anthony KR \& KE Fabricius. 2000. Shifting roles of heterotrophy and autotrophy in coral energetics under varying turbidity. Journal of Experimental Marine Biology and Ecology 252(2): 221-253.

Anderson K, M Pratchett \& A Baird. 2012. Summer growth rates of corals at Lord Howe Island, Australia. In: Proceedings of the 12th International Coral Reef Symposium, Cairns, QLD, Australia, Panama, pp. 9-13.

Bandaranayake WM. 1998. Mycosporines: are they nature's sunscreens? Natural Product Reports 15(2): 159-172.

Baums IB. 2008. A restoration genetic guide for coral reef conservation. Molecular Ecology 17: 2796-2811.

Cunning R, PW Glynn \& AC Baker. 2013. Flexible associations between Pocillopora corals and Symbiodinium limit utility of symbiosis ecology in defining species. Coral Reefs 32(3), 795-801.

Chavanich S, V Viyakarn, T Loyjiw, P Pattaratamrong \& A Chankong. 2009. Mass bleaching of soft coral, Sarcophyton spp. in Thailand and the role of temperature and salinity stress. ICES Journal of Marine Science 66(7): 1515-1519.
Edwards AJ \& ED Gomez. 2007. Reef restoration concepts and guidelines: making sensible management choices in the face of uncertainty. Capacity Building for Management Programme/Coral Reef Targeted Research, St. Lucia, 38 pp.

Fabricius KE. 2005. Effects of terrestrial runoff on the ecology of corals and coral reefs: review and synthesis. Marine Pollution Bulletin 50(2): 125-146.

Figueroa-Camacho AGF \& H Nava. 2016. Rehabilitación de la cobertura de corales del género Pocillopora (Lamarck 1816) usando una técnica adaptada a hábitats rocosos sublitorales. Biológicas Revista de la DES Ciencias Biológico Agropecuarias Universidad Michoacana de San Nicolás de Hidalgo 17(1): 31-36.

Forrester GE, K Taylor, S Schofield \& A Maynard. 2012. Colony growth of corals transplanted for restoration depends on their site of origin and environmental factors. Marine Ecology 34(2): 186-192.

Galván-Villa C M, E López-Uriarte \& J Arreola-Robles. 2011. Diversidad, estructura y variación temporal del ensamble de peces asociados al arrecife coralino de Playa Mora, Bahía de Tenacatita, México. Hidrobiológica 21(2): 135-146.

Hauri C, K Fabricius, B Schaffelke \& C Humphrey. 2010. Chemical and physical environmental conditions underneath mat-and canopy-forming macroalgae, and their effects on understorey corals. PLoS ONE 5(9): e12685. < https://doi.org/ 10.1371/journal.pone.0012685>

Hill J \& CLIVE Wilkinson. 2004. Methods for ecological monitoring of coral reefs, $117 \mathrm{pp}$. Australian Institute of Marine Science, Townsville.

Hunt J \& W Sharp. 2014. Developing a comprehensive strategy for coral restoration for Florida. State Wildlife Grant Award T-32-R 1169, Final Report. <https:// www.st.nmfs.noaa.gov/coralrestoration/pdf/ Hunt\%20and\%20Sharp\%202014.pdf>

Johannes RE \& WJ Wiebe. 1970. Method for determination of Coral tissue biomass and composition. Limnology and Oceanography 15(5): 822-824.

Karp GC. 2010. Cell and molecular biology: Concepts and experiments, 776 pp. John Wiley \& Sons, Gainesville.

Korbee N, FL Figueroa \& J Aguilera. 2006. Acumulación de aminoácidos tipo micosporina (MAAs): biosíntesis, fotocontrol y funciones ecofisiológicas. Revista Chilena de Historia Natural 79: 119-132.

Liñán-Rico A \& Y Cárdenas-Conejo. 2013. Use of molecular techniques to study the ecology of microorganisms associated with corals. In: Liñán-Cabello M (ed). Corals: Classification, habitat and ecological significance 1: 1-20. Nova Science Publishers, New York.

Liñán-Cabello MA, LA Flores-Ramírez, DMG Cobo, ST Zenteno, M Olguín, O Olivos \& AT Gómez. 2009. Response to short term ultraviolet stress in the reef-building coral Pocillopora capitata (Anthozoa: Scleractinia). Revista de Biología Tropical 58: 103-118. 
Liñán-Cabello MA, ELA Flores, ST Zenteno, MN Olguín, RA Sosa, MB Patiño \& OA Olivos. 2010. Seasonal changes of antioxidant and oxidative parameters in the coral Pocillopora capitata on the Pacific coast of Mexico. Marine Ecology 31(3): 407-417.

Liñán-Cabello MA, LA Flores-Ramírez, MA LaurelSandoval, E Mendoza-García, OS Santiago \& MA Delgadillo-Nuño. 2011. Acclimation in Pocillopora spp. during a coral restoration program in Carrizales Bay, Colima, Mexico. Marine and Freshwater Behaviour and Physiology 44(1): 61-72.

Liñán-Cabello MA, A Olivos-Ortiz, S Quijano-Scheggia, D Muñiz-Anguiano, ML Reséndiz-Flores \& CH Ortega-Ortiz. 2016. Effects of terrestrial runoff on the coral communities in Santiago Bay, Colima, Mexican Pacific Coast. Revista de Biologia Tropical 64(3): 1185-1200. <doi: http://dx.doi.org/10.15517/rbt.v64i3.21817>

Liu G, JL Rauenzahn, SF Heron, CM Eakin, WJ Skirving, T Christensen \& J Li. 2013. NOAA Coral Reef Watch $50 \mathrm{~km}$ satellite sea surface temperature-based decision support system for coral bleaching management. NOAA Technical Report NESDIS 143: 1-33. <https:// data.nodc.noaa.gov/coris/library/NOAA/CRCP/project/ $20370 / \mathrm{NOA}$ A C R W $50 \mathrm{~km} \mathrm{~S}$ a t S S Te m p NESDISTechReport143.pdf>

López-Pérez RA, LE Calderón-Aguilera, H Reyes-Bonilla, JD Carriquiry, P Medina-Rosas, AL Cupul-Magaña \& BM Luna-Salguero. 2012. Coral communities and reefs from Guerrero, Southern Mexican Pacific. Marine Ecology 33(4): 407-416.

Mayfield AB, L-H Wang, P-C Tang, T-Y Fan, T Yi-Yuong, L Ching-Lin \& C-S Chen. 2011. Assessing the impacts of experimentally elevated temperature on the biological composition and molecular chaperone gene expression of a reef coral. PLoS One 6(10): e26529. <https://doi.org/10.1371/ journal.pone.0026529>

Mayfield AB, T-Y Fan \& C-S Chen. 2013. Physiological acclimation to elevated temperature in a reef-building coral from an upwelling environment. Coral Reefs 32(4): 909-921.

Miller MW \& ME Hay. 1998. Effects of fish predation and seaweed competition on the survival and growth of corals. Oecologia 113: 231-238.

Obura D. 2009. Reef corals bleach to resist stress. Marine Pollution Bulletin 58(2): 206-212.

Pandolfi JM, SR Connolly, DJ Marshall \& AL Cohen. 2011. Projecting coral reef futures under global warming and ocean acidification. Science 333(6041): 418-422.
Prahl H \& B Vargas-Angel. 1990. Tasa de crecimiento del coral Pocillopora damicornis en un arrecife costero del Pacífico Colombiano. Revista de Ciencias 2: 37-43.

Reyes-Bonilla H, LE Escobosa-González, AL CupulMagaña, P Medina-Rosas \& LE Calderón-Aguilera. 2013. Estructura comunitaria de corales zooxantelados (Anthozoa: Scleractinia) en el arrecife coralino de Carrizales, Pacífico Mexicano. Revista de Biología Tropical 61(2): 583594.

Seveso D, S Montano, G Strona, I Orlandi, M Vai \& P Galli. 2012. Up-regulation of Hsp60 in response to skeleton eroding band disease but not by algal overgrowth in the scleractinian coral Acropora muricata. Marine Environmental Research 78:34-39.

Shick JM, WC Dunlap, BE Chalker, AT Banaszak \& TK Rosenzweig. 1992. Survey of ultraviolet radiation-absorbing mycosporine-like amino acids in organs of coral reef holothuroids. Marine Ecology Progress Series 90(2): 139148.

Siebeck UE, D Logan \& NJ Marshall. 2008. CoralWatch: A flexible coral bleaching monitoring tool for you and your group. Proceedings of the 11th International Coral Reef Symposium, Ft. Lauderdale, Florida, 7-11 July 2008, Vol. 1: 549-553.

Siebeck UE, N Marshall, A Klüter \& O Hoegh-Guldberg. 2006. Monitoring coral bleaching using a colour reference card. Coral Reefs 25(3): 453-460.

Smith JE, CL Hunter \& CM Smith. 2010. The effects of top-down versus bottom-up control on benthic coral reef community structure. Oecologia 163(2): 497-507.

Strickland JDH \& TR Parsons. 1972. Determination of reactive silicate. In: A practical handbook of seawater analysis. Fisheries Research Board of Canada, Bulletin 167: 65-70.

Szmant AM. 2002. Nutrient enrichment on coral reefs: Is it a major cause of coral reef decline? Estuaries 25(4B): 743766.

Tortolero-Langarica JJA, AL Cupul-Magaña \& AP Rodríguez-Troncoso. 2014. Restoration of a degraded coral reef using a natural remediation process: A case study from a Central Mexican Pacific National Park. Ocean \& Coastal Management 96: 12-19.

Yeemin T, M Sutthacheep \& R Pettongma. 2006. Coral reef restoration projects in Thailand. Ocean \& Coastal Management 49(9): 562-575. 